\title{
Las agriculturas territorializadas, oportunidades y retos frente al paradigma agroindustrial
}

\author{
Susana Ramírez-García
}

Universidad Complutense de Madrid. Departamento de Geografía Humana susamaranta@yahoo.es

\section{Olga Inmaculada Mancha-Cáceres}

Universidad Complutense de Madrid. Departamento de Antropología Social omancha@ucm.es

\section{Consuelo Del-Canto-Fresno}

Universidad Complutense de Madrid. Departamento de Geografía Humana ccantofr@ucm.es

\section{Resumen}

Este artículo se plantea dos objetivos. El primero es interrogarse sobre el papel que desempeñan hoy las agriculturas locales en el desarrollo sostenible de los espacios rurales y en qué medida construyen un movimiento de retorno de la agricultura al territorio que propicia nuevas relaciones entre ambos, expresadas en maneras diferentes de producir, en nuevos objetivos y nuevos lazos con el territorio. En segundo lugar, se busca reflexionar sobre la difícil convivencia de dos paradigmas relativos a la producción agroalimentaria, el industrial hipermoderno, basado en la globalización/mundialización, y el posmoderno, basado en el anclaje territorial.

Palabras clave: agricultura local; circuitos cortos alimentarios; sellos de calidad certificada; SIAL; agroecología

Resum. Les agricultures territorialitzades, oportunitats $i$ reptes davant del paradigma agroindustrial

Aquest article es planteja dos objectius. El primer és interrogar-se sobre el paper que exerceixen avui les agricultures locals en el desenvolupament sostenible dels espais rurals i en quina mesura construeixen un moviment de tornada de l'agricultura al territori que propicia noves relacions entre tots dos, expressades en maneres diferents de produir, en nous objectius i nous llaços amb el territori. En segon lloc, es reflexiona al voltant de la difícil convivència entre dos paradigmes vinculats a la producció agroalimentària, l'industrial hipermodern, basat en la globalització/mundialització, i el postmodern, basat en l'ancoratge territorial.

Paraules clau: agricultura local; circuits curts alimentaris; segells de qualitat certificada; SIAL; agroecologia 
Résumé. La "territorialisation" de l'agriculture: Opportunités et défis face au modèle agroindustriel

Cet article se propose deux objectifs. En premier lieu, il s'interroge sur le rôle des agricultures locales dans le développement durable des espaces ruraux et dans quelle mesure ces dernières participent au mouvement de retour à la terre et engendrent des relations nouvelles entre agriculture et territoires, avec des nouvelles manières de produire, de nouveaux objectifs et de nouvelles interactions avec le territoire. En second lieu, l'article réfléchit sur la difficile cohabitation entre ces deux modèles de production agroalimentaire: le modèle industriel hypermoderne qui s'intègre dans les procès de globalisation-mondialisation, et le modèle post-moderne qui se fonde sur l'ancrage territorial.

Mots-clés: agriculture locale; circuits courts alimentaires; signes de qualité certifiés; SIAL; agroécologie

\section{Abstract. Territorialised agriculture: opportunities and challenges facing the agro-industrial paradigm}

This paper has two main objectives: to examine the role played by local agriculture in the sustainable development of rural areas; and to investigate their involvement in the tendency of agriculture to return to the territory, which promotes new links between both, expressed through different production practices, new objectives and a stronger relationship. The paper also aims to consider the difficult coexistence of two paradigms related to agri-food production: the hypermodern industry, based on globalisation, and the postmodern, based on territorial embeddedness.

Keywords: local agriculture; short food chains; certified quality labels; localized agro-food systems; agroecology

\section{Sumario}

1. Introducción

2. Las agriculturas locales en el desarrollo sostenible de los espacios rurales

3. La comprometida convivencia entre agriculturas locales y agroindustria
4. Algunas estrategias de territorialización de la actividad agraria y agroalimentaria

5. Conclusiones

Referencias bibliográficas

\section{Introducción}

Este artículo se interroga sobre el papel que desempeñan las agriculturas locales en el desarrollo sostenible de los espacios rurales y en qué medida construyen un movimiento de retorno de la agricultura al territorio que propicia nuevas relaciones entre ambos. Un movimiento que para algunos autores constituye un nuevo paradigma enfrentado a la industrialización agroalimentaria a todas luces dominante. Un segundo objetivo del artículo consiste en reflexionar sobre la difícil convivencia entre dos paradigmas: el industrial hipermoderno, 
basado en la globalización/mundialización, y el posmoderno, basado en el anclaje territorial. Este último, denominado por Wiskerke (2009) paradigma agroalimentario territorial integrado, se fundamenta en " "estilos de agricultura" vinculados a la agricultura familiar y campesina (Ploeg, 2003, 2008) y a estrategias multifuncionales de diversificación productiva (Renting et al., 2009) enmarcados en un nuevo proceso de modernización ecológica (Marsden, 2004) y posproductivismo (Ilbery y Bowler, 1998, Shuckmith, 1993)» (Soler y Calle, 2010: 262).

Para ilustrar algunos de los elementos que representan este paradigma alternativo se analiza el papel que desempeñan en la territorialización agroalimentaria tres estrategias presentes en los espacios rurales europeos. Los productos locales, identificado su origen con sellos oficiales como denominación de origen protegida - DOP — o indicación geográfica protegida - IGP-, las redes alimentarias alternativas y canales cortos de comercialización (CCC), a los que se halla estrechamente vinculada la agroecología, y los sistemas agroalimentarios localizados (SIAL), como concentración local de pequeñas empresas agroalimentarias.

Los resultados y conclusiones expuestos son fruto de los diferentes proyectos de investigación y las reflexiones que el grupo de investigación GECO$\mathrm{MED}^{1}$ viene realizando en los últimos diez años en el centro peninsular, asentados sobre una metodología constructivista que integra la subjetividad de los actores socioeconómicos sobre los que se investiga. Se trata aquí de hacer una síntesis de las principales líneas exploratorias del grupo apoyada en otros estudios de caso (de distribución mundial) recogidos en una amplia revisión bibliográfica.

\section{Las agriculturas locales en el desarrollo sostenible de los espacios rurales}

Para evaluar el peso de las agriculturas industriales se puede hacer uso de análisis numéricos sobre sus volúmenes, superficies o mercados que poco reflejan la realidad territorial que soporta la actividad. Sin embargo, al centrar el interés en las agriculturas locales, son otras las variables que cobran importancia.

En primer lugar, para analizar estas agriculturas se adopta lo local como escala siguiendo un enfoque territorial, donde el territorio es entendido como una construcción social y como el espacio político de la acción social. Es

1. Entre otros y directamente relacionados con las conclusiones de este artículo: «Sistemas productivos locales en el sector vitivinícola madrileño: una opción para el desarrollo rural», financiado, en concurrencia competitiva, por la Consejería de Educación de la Comunidad de Madrid; «El sistema de producción localizado de leche y derivados en la Comunidad de Madrid», por encargo de la Consejería de Economía e Innovación de la Comunidad de Madrid; «La denominación de origen "Aceite de Madrid": ¿un sistema agroalimentario localizado?», financiado, en concurrencia competitiva, por la Consejería de Educación de la Comunidad de Madrid, y "Circuitos cortos de alimentación: innovaciones sociales para la conformación de nuevos estilos de vida sostenibles y la articulación territorial en el centro peninsular», financiado, en concurrencia competitiva, por el Ministerio de Economía y Competitividad. 
el lugar donde se fragua un nuevo paradigma de gestión pública, de desarrollo institucional y de participación social, y donde es posible generar ventajas competitivas a partir de sus recursos específicos (Mollard, 2001; Marsden y Sonnino, 2008). Estos recursos pueden ser reconsiderados a raíz de una transformación coyuntural (como la actual crisis económica o el auge de la demanda de productos locales) y sirven de fundamento para la conformación de nuevas ruralidades.

Sobre estas premisas, parece pertinente hablar de la territorialización de la agricultura al interrogarse sobre el papel de las agriculturas locales en el desarrollo sostenible. Para ello se tiene en cuenta la teoría de la reestructuración rural, o la nueva ruralidad (Pérez, 2001, Schejtman y Berdegué, 2004, De Janvry y Sadoulet, 2005), nacidas para explicar el escenario complejo de una ruralidad sujeta a transformaciones funcionales de gran impacto en el territorio, cuyo significado está ligado en lo económico al posproductivismo, en lo social a la posmodernidad y en lo político al desarrollo rural.

En lo concreto, la derrota que ha tomado la producción y comercialización agroalimentaria a partir de mediados del siglo xx ha conducido a un doble desarraigo. Por una parte, la producción agraria globalizada e industrializada ha perdido su especificidad local y su vínculo sociocultural. Por otra parte, el consumidor también ha perdido su vinculación cultural y estacional con el alimento. Contra esta corriente dominante, en las últimas décadas han ido apareciendo, y creciendo en visibilidad e implantación, diferentes fórmulas de agricultura local que parecen responder a un proceso de «reterritorialización», entendiendo la territorialización como el estado en el que se combinan en la población sentimientos de pertenencia, apropiación e identidad colectivas, en este caso vinculadas al producto, su modo de producción y la localidad originaria.

Una reterritorialización que parece tener dos orígenes principales. Por una parte, la investigación-acción en desarrollo rural ligado a lo agrario, pionera en estas metodologías (López García, 2012: 78), ha tratado, principalmente en países pobres, de promover procesos de revalorización de la diversidad local y de aprovechamiento de los recursos locales específicos. Por otra parte, en los países más ricos esta promoción parece haber surgido de la iniciativa de sectores sociales que tratan de responder a coyunturas de crisis de diverso tipo: económicas, identitarias, ambientales, sociales. Desde la perspectiva del consumo, Acampora y Fonte (2007: 191) consideran que esta dinámica parte de la incorporación de "preocupaciones sociales y éticas en relación con la supervivencia de la comunidad y de las culturas locales, la protección del medio ambiente, la protección de la naturaleza, el bienestar de los animales. Los estilos de consumo se vuelven un elemento de construcción de las mismas identidades sociales (Maffessoli, 1996)». En la producción se encuentran, según Soler y Calle (2010: 261), respuestas individuales: «estrategias familiares de diversificación de ingresos mediante la pluriactividad y la agricultura a tiempo parcial, sistemas de manejo campesinos asociados a bajos insumos, diferenciación de sus productos como artesanales, naturales, ecológicos, venta directa o canales 
cortos de comercialización", y respuestas colectivas en alianza con el consumidor, que están creando nuevas de redes de abastecimiento caracterizadas «por una redefinición de los mecanismos de intercambio que implican, implícita o explícitamente, un cuestionamiento de los mercados competitivos».

Estas realidades expresan la construcción de un movimiento de retorno de la agricultura al territorio que, a pesar de su dimensión marginal o alternativa, revela ciertos cambios en determinados paisajes agrarios, se introduce en diferentes estratos sociales incluso a través de los medios de comunicación y comienza a formar un sustrato que puede servir de base para una transformación ambiental y socioproductiva en la que las iniciativas oficiales siguen estando un paso por detrás (MAGRAMA, 2012). Es un movimiento que hace hincapié en las novedades en la relación agricultura-territorio: nuevas metas, nuevas maneras de producir y nuevos lazos con los que afianzar la actividad (Mancha Cáceres y Ramírez García, 2014).

Junto con la obtención de ingresos, los nuevos, o renovados, productores dotan a su actividad de una significación social al marcarse como objetivos ofrecer un producto de calidad organoléptica, sostenible (ambiental, económica, cultural y socialmente) y con identidad. Estos objetivos inmanentes a las unidades de producción se pueden extrapolar, según la perspectiva adoptada, a lo local en busca de la resiliencia y el desarrollo territorial (Ciolos, 2012).

Alcanzar estas expectativas requiere nuevas maneras de producir que integran mecanismos y procesos para recuperar la identidad de los productos y los territorios, converger con el funcionamiento ecosistémico, hacer visible el valor de lo local y vender cultura local, de manera que se logra la rehabilitación de las agriculturas locales.

Estas prácticas crean nuevos lazos o «reconectan» procesos, espacios y dinámicas que se viven en el territorio: procesos de producción con procesos de consumo; espacios rurales o periurbanos con urbanos; dinámicas sociales vinculadas a los movimientos ciudadanos con dinámicas sociales de revalorización de la identidad de productos y territorios.

Con estas novedades se articula una economía de proximidad fundada en nuevas relaciones entre las zonas rurales y la agricultura, donde el anclaje territorial se hace prominente. Nuevas relaciones en las que se puede discernir entre los planos social, económico, cultural, ambiental y político. En lo social, la componente relacional recupera una fuerza mermada o pérdida, y busca un cambio social en respuesta a las crisis que deberá tener su correlato en el resto de los planos. En especial en el económico, donde se postula que la cooperación y la colaboración, junto con la revalorización de lo específico y el acercamiento directo al consumidor, permitirán obtener mejores rentas que las obtenidas individualmente. Reconsiderar el valor de lo local forma parte de una visión recuperadora de la cultura propia en gran medida asolada por el tsunami globalizador. El plano ambiental se considera en mayor o menor medida en las estrategias analizadas, y es una de las claves principales en los circuitos cortos, ya que se transforma en el leitmotiv en aquellas experiencias en que el plano político manifiesto es la transición agroecológica. Se busca la recuperación del 
equilibrio ecológico en los agroecosistemas aumentando la biodiversidad cultural y natural, reduciendo impactos y apostando por el decrecimiento como vía para alcanzar la sostenibilidad ampliada (González de Molina, 2012). Para Montasell et al. (2014: 654), "plantear la "proximidad" [...] es una respuesta a la seguridad alimentaria, a la reducción de la huella ecológica derivada del transporte de los productos, al cambio climático con la reducción del gasto energético, a la reducción de las emisiones de $\mathrm{CO}$ y otros gases con efecto invernadero, y al fomento y diversificación de la economía local».

La valorización de lo rural se inserta, así, en una nueva lógica económica en la que la competitividad no está basada en el productivismo y la maximización del beneficio no es el objetivo primordial —el precio es uno de los tantos mecanismos de coordinación de los mercados-, en la que el modelo productivo se puede calificar como "territorializado" y en la que se dan formas de reagrupamiento de actores para la organización de la producción del tipo distrito industrial, sistema productivo local (SPL) o su expresión específica para los productos agroalimentarios, los sistemas alimentarios localizados (SIAL).

En esta nueva lógica, la producción está orientada a la calidad o se basa en «activos específicos». La imagen positiva del patrimonio rural es un apoyo clave para esta reterritorialización, así como las ventas en los mercados locales y la proximidad del consumidor con respecto al producto. Esta cercanía se usa para promocionar una mayor variedad de bienes y servicios, a veces organizada como apuntan los estudios sobre los "cestos de bienes y servicios territoriales» y la teoría de la renta de calidad territorial (Pecqueur, 2001). Estos procesos dependen de la existencia de formas de conocimiento de lo local a través de las cuales los clientes mantienen un vínculo, al menos simbólicamente, con los rasgos culturales y regionales de los alimentos (Boulanger y Legouy, 2008).

Además, el paisaje agrario se convierte en "paisaje de la memoria», en lugar de arraigo territorial y de identidad. En resumen, mientras se somete a una crisis social y de identidad, la agricultura es parte de la cultura tradicional que debe ser preservada. A cambio, ella participa en la promoción de las zonas rurales, del atractivo de los territorios y de su imagen. Este «desarrollo del patrimonio», basado en estrategias de los actores, se centra cada vez más en el ámbito local.

\section{La comprometida convivencia entre agriculturas locales y agroindustria}

La controversia teórica entre la agricultura local y la agroindustria se puede centrar en la dialéctica territorialización/desterritorialización, dos movimientos contrapuestos entre la escala local y la global, entre las economías locales y la mundializada.

Según los distintos tipos de espacios rurales (clasificación que puede depender más de criterios de oportunidad que de determinación ambiental: disponibilidad de suelo, de mano de obra, interés de los grandes capitales...), las manifestaciones que en ellos se producen (asociadas fundamentalmente a características culturales e identitarias) y los comportamientos y disposición de empresas y trabajadores, la agricultura se desarrollará siguiendo las tenden- 
cias modernas, basadas en la globalización o mundialización, o posmodernas, basadas en el anclaje territorial.

Los procesos de vuelta al territorio parecen estar llevándose a cabo centrados en dos dimensiones. Por un lado, se apuesta por la valorización del terroir y la fuerza de lo local como espacio de movilización de actores públicos/privados, y por otro, se sitúa en un lugar central la importancia de la movilización colectiva en la puesta en marcha de proyectos que articulan todas las dimensiones del desarrollo territorial (Mancha Cáceres y Ramírez García, 2014; Del Canto Fresno y Ramírez García, 2009; Freitas Caetano et al., 2013).

La desterritorializacion de la agricultura viene determinada por la ruptura entre agricultura y territorio, lo que se podría calificar como el territorio desarraigado. El crecimiento de la agricultura industrial sucede como si los aspectos específicos del terroir y las "pequeñas áreas» tradicionales pasaran a contar muy poco, a perder utilidad y valor. El resultado final es la situación actual, con un sistema agroalimentario dominante intensivo, industrializado y desterritorializado.

En todos los casos, los factores arraigados a los territorios parecen tenues, los sentimientos de pertenencia y propiedad de los actores rurales, cada vez más diversificados, se debilitan, y podemos hablar de "desterritorialización» para una agricultura productivista que presta poca atención a las agriculturas campesinas, insertadas ahora en el sector agroindustrial y de las cuencas de producción especializadas.

De momento, el modelo productivo territorializado coexiste con el modelo moderno de productividad.

En palabras de Monllor (2013: 13):

la coexistencia de los dos modelos en el mismo espacio y tiempo crea «un campo de batalla complejo en el que diferentes intereses, perspectivas y proyectos tienen que competir» (Ploeg, 2008: 151). En este sentido, es interesante remarcar el papel estratégico de las personas que consumen alimentos, entendiendo que el poder de compra es infinito y que cada moneda dedicada al nuevo campesinado forma parte del cambio. El surgimiento y revitalización de las actividades agrarias estratégicas para el territorio están totalmente vinculados al compromiso que adquieren los consumidores y las consumidoras con el nuevo modelo agrario.

En este sentido, a la preocupación del consumidor por la calidad del producto viene dándosele respuesta a través de la identificación oficial de los signos de calidad y origen territorial (denominación de origen, indicación geográfica protegida, producto ecológico...). La adopción de estos etiquetados por las grandes corporaciones, más aún se podría decir su apropiación, al saturar el mercado de productos con estos distintivos, ha vuelto a hacer saltar las alarmas del consumidor preocupado por la calidad, el carácter saludable, la sostenibilidad y el origen de sus alimentos, y esta preocupación renovada es el origen de las redes alternativas, los circuitos cortos y la agroecología. Las ventajas de las dinámicas colectivas que pueden generan ambos procesos son también el 
fundamento de los estudios sobre sistemas agroalimentarios localizados, que constituyen otra estrategia de territorialización agraria.

La fuerza de las agriculturas alternativas frente a la dominancia globalizadora depende mucho de sus coyunturas. En América Latina, donde combaten pobreza y exclusión, algunos gobiernos (Perú, Ecuador, Bolivia) lo incluyen como parte de sus políticas (ejemplo: el apoyo a la compra pública) y comienzan a ejercer cierto contrapeso en relación con los intereses de las grandes corporaciones agroalimentarias tan fuertes en la región (Sabourin et al. 2014).

En Estados Unidos, De Longe et al. (2016: 272), analizando la financiación pública para investigación en agricultura, concluyen que estos fondos son escasos para el avance de la agroecología y los sistemas agroalimentarios sostenibles (entre los que incluyen aquellos de base social que podrían asimilarse a los aquí definidos como territorializados), y que es urgente una financiación pública adicional que ayude a mitigar los impactos ambientales y sociales generados por la agricultura industrial.

Si pensamos en Europa, el movimiento es más evidente y tiene más adeptos, sean productores o consumidores, cuanto más desarrollados sean los estados. Así, la preocupación por el planeta, por lo que comemos, por la salud, etc. parece propia de sociedades urbanas y desarrolladas. Es estos espacios se configura, además, una vertiente político-social minoritaria en los sectores militantes más radicales. En palabras de Calle et al. (2009: 2), las respuestas sociales a la desafección al sistema agroalimentario se pueden tipificar en tres clases: «nuevos estilos agroalimentarios, nuevos cultivos sociales y nuevos movimientos globales», todos ellos en busca de otro modelo de consumo y otro paradigma alimentario y político. González de Molina (2012) considera que el consumo agroalimentario sostenible no conseguirá significar un porcentaje relevante sin un marco institucional adecuado, a pesar de la pujanza de las acciones individuales y colectivas, y que esa situación no se recoge aún en las políticas europeas.

Más allá de las reflexiones teóricas, se dibuja un contexto real muy heterogéneo en el que, naturalmente, no existe una única respuesta sobre cómo se confrontan los dos paradigmas. Tratando de sistematizar las claves de una convivencia que pueda ser duradera entre los dos paradigmas, se dibujan los siguientes aspectos:

- La competitividad creciente y la expansión del mercado global: los mecanismos de que dispone la maquinaria global son suficientes para anular o al menos restringir a posiciones insignificantes las producciones y comercios que no participan de sus procesos y para reconducir las iniciativas alternativas hacia la inclusión en su esfera, como ha ocurrido con la agricultura ecológica certificada que ha sido integrada en la lógica productivista.

- El papel de las disposiciones legales, que pueden desatender o discriminar a los productores pequeños frente a la industria agroalimentaria o bien crear las condiciones para su coexistencia.

- La existencia de apoyos institucionales en forma de políticas, estrategias o programas que consideren positiva y favorezcan la diversificación de formas 
de producción en las que se integren las agriculturas territorializadas, así como la comercialización en circuitos de proximidad.

- Un abordaje público del problema de disponibilidad de tierras vinculado a los sistemas de propiedad, aprovechamiento y obtención de rentas subvencionadas.

- La conciencia de los productores de los aportes sociales de su actividad, que comprenden aspectos culturales, identitarios, ambientales y territoriales.

- La conciencia de los consumidores de la importancia de su elección y de sus repercusiones sociales, ambientales y territoriales.

- La percepción social de las tareas agrarias, en ocasiones muy infamadas, lo que limita la consideración del campo como salida profesional.

\section{Algunas estrategias de territorialización de la actividad agraria y agroalimentaria}

No todos los territorios se comportan del mismo modo ni en lo que respecta a la introducción de nuevas relaciones ni a sus consecuencias: como ejemplos, se pueden destacar la situación favorable del productor periurbano con respecto a su relación con el consumidor urbano, o sus desventajas en la disponibilidad de tierras en contraste con los rurales más aislados de los potenciales mercados de consumo, o las coyunturas diferenciales en la percepción social de los trabajos agrarios en países o ámbitos culturales distintos.

$\mathrm{Al}$ mismo tiempo, las estrategias analizadas tienen respuestas diferentes tanto en sus planteamientos como en sus adaptaciones contextuales. En este epígrafe se trata de analizar esta casuística. Para ello, en primer lugar, se tipifican en la tabla 1 las tres estrategias en función del peso que adquieren los diferentes planos relacionales en sus propuestas, como conclusión de los estudios de caso llevados a cabo durante los últimos diez años en el centro peninsular. A continuación, se sintetizan los principales aportes de múltiples experiencias estudiadas en el ámbito internacional, que matizan y amplían conclusiones preliminares obtenidas en los estudios locales que se han realizado.

La literatura revisada analiza infinidad de temas relacionados con los sistemas alimentarios locales y regionales desde diversos enfoques.

Tabla 1. Estimación cualitativa de los pesos relativos de las componentes relacionales en las nuevas agriculturas territorializadas

\begin{tabular}{lccccc}
\hline & \multicolumn{4}{c}{ Nuevas relaciones agricultura-territorio } \\
\cline { 2 - 5 } & Social & Económico & Cultural & Ambiental & Político \\
\hline Etiquetados oficiales & $*$ & $* \star *$ & ${ }^{* \star *}$ & & ${ }^{* *}$ \\
SIAL & $* *$ & $* *$ & $* * *$ & & ${ }^{* *}$ \\
Redes alternativas, circuitos cortos & $* *$ & $* *$ & $* * *$ & $* *$ & \\
y agroecología & & & & & \\
\hline
\end{tabular}

Fuente: elaboración propia. 
Desde la producción-consumición, destacan los trabajos en torno a la mejora de estos sistemas, aumentando viabilidad económica de productores o facilitando accesos de consumidores a alimentos cultivados localmente por pequeños y medianos productores. Muchos de estos trabajos son informes para el desarrollo de canales de comercialización con mayor estabilidad económica y viabilidad para pequeños y medianos productores, así como el suministro de alimentos frescos y de gran calidad a los consumidores (Ploeg et al., 2008; Pimbert, 2008). La sostenibilidad ambiental es otro aspecto desarrollado en la literatura desde múltiples vertientes. Destacan los análisis sobre el logro de la sostenibilidad a partir de los productos locales, los CCC o los SIAL.

Otra importante línea de investigación analiza desde las perspectivas de la economía y la ecología políticas la diversidad actual de regímenes alimenticios y desentraña sus características, dinámicas y vínculos con problemáticas globales, como el acceso a los alimentos, los modelos de producción, la seguridad alimentaria o el comercio de alimentos, así como sus implicaciones en la vida de comunidades y colectivos del norte y del sur (Bailey, 2011; Grupo ETC, 2009; Morgan et al., 2009; Ploeg, 2008; Pimbert, 2008, entre otros). Este tipo de trabajos se vincula con la finalización, hace veinte años, de la Ronda Uruguay del GATT y las negociaciones comerciales globales (con la formación de la $\mathrm{OMC}$ ), y en otros trabajos se destaca la emergencia de un nuevo régimen neoliberal global de los alimentos. McMichael (2002) señala la implantación progresiva de un régimen corporativo alimentario industrial, apoyado y sostenido por los gobiernos neoliberales y el capital corporativo transnacional. Denominó a este nuevo régimen «food from nowhere» (alimentos de ningún lugar), caracterizado por la producción industrial y el comercio a gran escala de alimentos o materias primas para la industria alimentaria.

Desde la Academia, los movimientos sociales y la militancia agroalimentaria se señala la necesidad de poner en marcha alternativas basadas en las condiciones culturales y ecológicas locales como forma de oponerse a este régimen emergente, comenzando a fomentar y reconocer los sistemas alimentarios locales, la producción local, la denominada slow food o los huertos urbanos o comunitarios (Vivas, 2014).

Este análisis representa la política mundial de alimentos en torno a dos polos: uno globalizador, industrial, corporativo y capitalista, representado por los «alimentos de ningún lugar» de McMichael, y otro polo integrado, sostenible y local. El factor espacio está presente en ambos modelos de manera opuesta: en el primero, el alimento está "deslocalizado", mientras que en el segundo modelo, que incluiría los productos locales, los CCC y los SIAL, el lugar es un valor añadido fundamental, su elemento distintivo frente a la homogeneización. Esta visión dicotómica viene siendo cuestionada por investigadores como Manhire et al. (2012), para quienes existe un conjunto de alimentos a los que denominan «alimentos de algún lugar» que se mueven en lo global, pero que se presentan como sostenibles, ecológicos o locales: alimentos y productos locales, ecológicos, de comercio justo o con certificación de producción ecológica; involucran a grandes explotaciones y corporaciones agrícolas, a redes de comercio global y 
minorista, a entidades certificadoras y a mercados exclusivos de los países ricos. Son alimentos que comparten cualidades de los alimentos locales (más integrados cultural y ecológicamente), pero insertos en dinámicas globalizadas (cotizan en el mundo corporativo globalizado). En este sentido, examinan las dinámicas sociales y ecológicas de la categoría «alimentos de algún lugar», su potencial transformador de los modelos de desarrollo rural, así como sus efectos en la sostenibilidad ambiental. Para ello, analizan productos emblemáticos de la agricultura de exportación neozelandesa, como los kiwis, las manzanas, el vino y la lana merina, para ver cómo, en los últimos quince años, el ecoetiquetado ha transformado significativamente la industria agroalimentaria, y concluyen que los efectos sobre la sostenibilidad del etiquetado ecológico son muy diversos y permiten oportunidades moderadas para cambiar las prácticas sociales y los efectos ecológicos, pero no un cambio radical de las prácticas agrícolas.

\subsection{Producto local}

Siguiendo a Friedmann y McMichael (1989), el producto local se caracteriza por su elaboración a pequeña escala; está local y ecológicamente conectado; está impulsado por movimientos sociales, locales, de productores, por pequeños negocios o por la política local; está inserto cultural y socialmente en su contexto; su comercio se basa en sus «cualidades»; y es floreciente.

Producto y lugar están conectados de forma inseparable y ello garantiza la calidad del producto y su valoración social. El producto reúne en sí mismo las características del terroir, entendido como la tierra, el suelo y el lugar concreto del que procede. Es esta "promesa del lugar» del producto local (Bowen, 2015: 2) la que hace percibir los alimentos tradicionales como alternativas, políticamente destacables y económicamente rentables, a la homogeneización de la comida globalizada. Frente a la «comida de ningún sitio», la "comida de alguna parte» se caracteriza por ocupar nichos de consumo y producción saludables y sistemas complejos de auditoría e inspección, que van desde certificaciones ecológicas hasta modelos de comercio justo. Este conjunto de relaciones opera a escala mundial y hace visibles las condiciones locales de producción (Campbell, 2009: 312) a distancias de escala global.

Ya desde la época clásica, encontramos en el espacio mediterráneo toda una serie de marcas famosas asociadas a productos con un origen geográfico concreto: el vino de la isla griega de Quíos, el garo de Cartago Nova, las pasas de Corinto, los dátiles de Egipto y Palestina, los cabritos de Ambracia, las ostras de Bríndisi y del lago Lucrino eran muy valorados en la antigua Grecia y en Roma (Adam, 1828).

La cultura del terroir en Francia tiene hondas raíces y se encuentran referencias al mismo desde el siglo Xvi (Trubek, 2009)2 . En los primeros años del

2. La antropóloga Amy Trubek cita en su obra The Taste of Place: A Cultural Journey into Terroir (2009) a Oliver de Serres (1539-1619), Pierre Jean-Baptiste Le Grand d'Aussy (1737-1800) y Jean-Anthelme Brillat-Savarin (1755-1826) entre los autores que hablaron de la relación entre sabores y origen de los productos. 
siglo $\mathrm{xx}$, un grupo conformado por periodistas, cocineros famosos y escritores de libros de cocina, así como por productores diversos empieza a desarrollar la noción de terroir. Pronto se plantea la necesidad de proteger los productos y delimitar las prácticas productivas y los valores que entrañan con objeto de que no desaparezcan.

El primer antecedente en Francia de una IGP es la del queso de Roquefort (s. XVI). Otro ejemplo para proteger y asegurar la calidad del vino de Oporto es la creación, en 1756, de la Compañía General de la Agricultura de las Viñas del Alto Duero. Cien años más tarde se dan los primeros pasos para crear la denominación de origen (DO) vino de Burdeos, por iniciativa de los corredores de vino de la ciudad.

El concepto y figura legal DO se creó en Francia en el siglo xix y se extendió a todo el mundo especialmente desde los años noventa del siglo pasado. Parte de la vinculación entre producto y lugar y de la creencia de que las peculiaridades de un territorio se trasladan a los alimentos que en ellos se producen, por lo que deben protegerse, tanto los alimentos producidos como los diferentes componentes y soportes, materiales e inmateriales, que confluyen en su producción.

La Convención de París de Propiedad Intelectual, de 1883, y el Acuerdo de Madrid para la Represión de las Indicaciones Falsas o Engañosas sobre el Origen de los Bienes, de 1891, fueron las primeras iniciativas de tipo legal puestas en marcha para proteger los productos locales. Tras ambas disposiciones multilaterales para aumentar la protección de las indicaciones de procedencia y las DO, aparecieron en Francia la primera appellation d'origine en 1905 (Champagne) y la primera appellation d'origine contrôlée en 1935, que ampliaba el control sobre el proceso de elaboración. En España, siguiendo el modelo francés, se crearon las primeras DO en 1932 (diecinueve DO reconocidas de forma simultánea). Posteriormente, el Acuerdo de Lisboa de 1958 representa el mayor acuerdo multilateral de DO hasta la fecha, suscrito por un total de 28 países.

A partir de los años noventa, el terroir, la revalorización y la patrimonialización de los productos locales comienzan a extenderse a otros espacios del planeta, de manera paralela a la globalización y la estandarización de la industria alimentaria. Desde 1994, Estados Unidos y otros países argumentan, en las negociaciones de comercio multilaterales y bilaterales, que las figuras de protección atentan contra la libertad de comercio. Estos países perciben las DO como políticas proteccionistas.

Desde Francia, una diversidad de actores, productores, intelectuales, consumidores urbanos, organizaciones agrícolas, etc. se han opuesto a la homogeneización y estandarización de la comida, y han hecho que el terroir incorpore, a sus elementos identitarios, componentes de reivindicación y lucha política y social, necesarios ahora para preservar los símbolos de tradición y continuidad asociados al campesinado.

A estos movimientos se ha unido recientemente la estrategia discursiva de la Unión Europea, que defiende las DO como estrategia de desarrollo rural 
válida no solo para los agricultores europeos, sino también para los del resto del mundo (Kerr, 2006: 7).

\subsection{Canales cortos de comercialización}

Ante la diversidad de sistemas de producción y comercialización de alimentos locales es difícil encontrar una única definición para canal o circuito corto de comercialización. En la literatura figuran definiciones en las que los CCC se restringen a aquellos en los que los únicos agentes involucrados son el productor y el consumidor, y definiciones más amplias, como la recogida en el Artículo 2 de la Propuesta de Reglamento del Parlamento Europeo y del Consejo sobre la ayuda al desarrollo rural a través del Fondo Europeo Agrícola de Desarrollo Rural (Feader): «cadena de suministro formada por un número limitado de agentes económicos, comprometidos con la cooperación, el desarrollo económico local y las relaciones socioeconómicas entre productores y consumidores en un ámbito geográfico cercano».

Aunque la venta directa siempre ha existido, el CC aparece como movimiento en 1965 cuando, en Japón, un grupo de madres reaccionan ante la creciente industrialización de la agricultura y el uso masivo de pesticidas y herbicidas y fundan las primeras alianzas (teikis) con agricultores. El campesino se comprometía a producir alimentos sin recurrir a la química y las familias suscribían la compra de la cosecha. En estos años aparecerán también en Europa modelos similares, como los Gruppi di Acquisto Solidale italianos, los Landwirtschaftsgemeinschaftshof alemanes o las associations alimentaires suizas. La idea cruzará el Atlántico y en Canadá y en los Estados Unidos se crea el modelo de Community Supported Agriculture. El caso francés de la Association pour le Maintien de l'Agriculture Paysanne (AMAP) surge en 2002.

Asimismo, los CCC comienzan a despegar en numerosos países de América Latina y el Caribe, como México, Brasil, Ecuador y Perú, con la creación de innumerables mercados ecológicos u orgánicos (como los de Loja y Cuenca en Ecuador, o Jalisco y Xalapa en México) o el establecimiento de compras públicas de alimentos para suministro de escuelas (Programa Hambre Cero de Brasil) (Romero, 2014).

El modelo inicial ha ido transformándose a medida que incorpora nuevos elementos que, según Milone (2014), explican su proliferación, como el impacto del cambio climático, el desafío de la seguridad alimentaria, la escasez de agua y el surgimiento de la pobreza y del desempleo como grandes problemas que condicionan la estrategia de desarrollo. También se suman otros fenómenos recientes vinculados a la agricultura, como la dependencia de los productores de los mercados globales y la disminución de la rentabilidad de la producción agrícola (bajada de precios y aumento de costes). Este último afecta de manera diferenciada a las fincas de la agricultura familiar y a las altamente tecnologizadas. Los modelos de producción agroecológicos tienen mayor capacidad de adaptación a los vaivenes de precios y de innovación productiva que los modelos altamente especializados y pueden idear nuevas actividades, 
Tabla 2. Modelos de canales cortos de comercialización

\begin{tabular}{|c|c|c|c|}
\hline Modelo de CCC & $\begin{array}{l}\text { Localización } \\
\text { (tiempo, espacio) }\end{array}$ & Referencias & Objetivos \\
\hline Teikeis & Japón, 1965 & $\begin{array}{l}\text { linuma (1995), } \\
\text { Fukuoka (1978), } \\
\text { Muramoto et al., } 2010\end{array}$ & \multirow{2}{*}{$\begin{array}{l}\text { Compra-venta directa bus- } \\
\text { cando producto sano y de } \\
\text { calidad y pagar al productor } \\
\text { un precio justo por su pro- } \\
\text { ducto. }\end{array}$} \\
\hline $\begin{array}{l}\text { Community } \\
\text { supported } \\
\text { agriculture (CSA) }\end{array}$ & EE. UU., años sesenta & Kneafsey et al. (2008) & \\
\hline Cestas & $\begin{array}{l}\text { Europa (cronología } \\
\text { variable, desde los años } \\
\text { ochenta en el Reino Unido } \\
\text { y los países nórdicos) }\end{array}$ & Torjusen et al. (2008) & $\begin{array}{l}\text { Compra-venta directa } \\
\text { acompañada de conciencia } \\
\text { del sistema alimenticio, } \\
\text { comunicación y relaciones } \\
\text { sociales y nueva manera de } \\
\text { consumir alimentos. Este } \\
\text { modelo invita a la gente a } \\
\text { involucrarse en la comida de } \\
\text { varias maneras: adquirien- } \\
\text { do conocimiento sobre el } \\
\text { sistema alimentario mundial } \\
\text { y concienciación política } \\
\text { (información, boletines); } \\
\text { experiencias sensoriales } \\
\text { (sentir la comida, caminar } \\
\text { los campos); las relaciones } \\
\text { sociales y la empatía (preo- } \\
\text { cuparse por los demás en el } \\
\text { sistema alimentario). }\end{array}$ \\
\hline $\begin{array}{l}\text { Mercados } \\
\text { ecológicos, } \\
\text { tianguis }\end{array}$ & $\begin{array}{l}\text { Europa, } \\
\text { América del Norte } \\
\text { y América del Sur }\end{array}$ & Romero (2014) & $\begin{array}{l}\text { Compra-venta directa } \\
\text { acompañada de la promo- } \\
\text { ción de vínculos entre pro- } \\
\text { ductores y consumidores. }\end{array}$ \\
\hline
\end{tabular}

Fuente: elaboración propia.

productos y mercados, así como nuevas formas de gestión y funcionamiento, como cooperativas, ventas en línea, venta directa en mercados locales, tiendas $\mathrm{y}$ restaurantes, etc.

Van der Ploeg analiza estas nuevas realidades emergentes desde un «nuevo paradigma del desarrollo rural», que requiere que la agricultura rescate aquellos valores que ha ido perdiendo al ser absorbida por el capitalismo y que son la esencia de una manera de ser y hacer campesina: coproducción, base de recursos autocontrolada, ordenación de las relaciones con los mercados que aseguren autonomía, supervivencia, fortalecimiento de la base de recursos, reducción de la dependencia, lucha por la autonomía, pluriactividad y modelos de cooperación.

El planteamiento de Ploeg (2008; 2010) y de Ploeg y Marsden (2008) incorpora el elemento político y de justicia social que no tenía la propuesta de cambio de paradigma en el mundo rural que expuso en 2006 la OCDE, que 
se basaba en la importancia del lugar frente a los sectores, las inversiones frente a las subvenciones y la articulación del territorio con una nueva gobernanza rural sin cuestionar la desigualitaria inserción de la agricultura y sus actores en el sistema económico capitalista global.

\subsection{Sistemas agroalimentarios localizados}

Para el IICA, «el término SIAL surge de una serie de trabajos, conducidos por el CIRAD, sobre pequeñas y medianas empresas agroalimentarias en África (López y Muchnik, 1997) y agroindustrias rurales (AIR) en Latinoamérica (Boucher y Muchnik, 1998)» en torno a la problemática de la alimentación de las poblaciones urbanas «y la valoración de los recursos locales como una posibilidad de generar valor agregado para la agricultura familiar, mediante el procesamiento de sus productos (GIS-SIAL, 2009)». Estos trabajos constatan la presencia de concentraciones locales de AIR especializadas, y de redes verticales y horizontales que estructuraban las cadenas agroalimentarias, favorecedoras de la movilización de recursos locales, tales como el saber hacer, la tecnología, la reputación y las condiciones ecológicas locales, los cuales, a su vez, eran elementos clave para propiciar dinámicas de desarrollo local (IICA, 2013: 15).

La noción de SIAL pretende cambiar la manera de pensar la producción y el consumo de alimentos partiendo de la valorización de los recursos territoriales, las problemáticas medioambientales y la importancia de las culturas alimentarias de las sociedades. Se definen como una forma de organización y un proceso de desarrollo local basado en una concentración geográfica de unidades agroalimentarias (explotaciones agrícolas; empresas agroalimentarias; empresas de servicios, de comercialización y de restauración, entre otras) asociadas por sus características y su funcionamiento a un territorio específico (Muchnik y Sautier, 1998). También son un movimiento colectivo de asociaciones de productores o de consumidores, de sindicatos agrarios, de organizaciones de apoyo o de redes de investigación que busca promover o defender una alternativa al sistema agroindustrial alimentario (Colonna et al., 2013). Así, se han constituido varias redes, tales como la Red SIALAM y la Red SIAL en Argentina y en México.

El origen de los SIAL entronca con las teorías de Marshall sobre los distritos industriales. Al elemento identitario territorial común (Beccatini, 1979; Cappechi, 1987) se añaden en el análisis otros elementos, como las externalidades, las redes sociales y la confianza entre actores. Además de la cooperación, la competencia entre estos actores también emerge como un factor decisivo del desarrollo territorial. En las siguientes décadas los conceptos de SPL (Courlet et al., 1993) y clúster (Schmitz, 1997; Porter, 1998) se consolidan a partir de estas primeras teorías explicativas.

\subsection{Contribución al anclaje territorial y perspectivas del paradigma alternativo}

Los modelos de conexión entre producto y lugar que hemos revisado, basados en los productos locales y el etiquetado diferenciado, los SIAL y las redes alter- 
nativas planteadas desde los CC y la agroecología, comparten formas de anclaje territorial. Todos ellos reconocen la importancia para su existencia del trabajo colectivo y de la imbricación de los múltiples actores en juego.

Por otro lado, los PL, los CCC y los SIAL basan su anclaje territorial en torno a factores específicos. Así, desde el lado de los productos del lugar, vemos cómo diferentes estudios de caso concluyen que las DO tienen efectos positivos en la actividad agrícola ${ }^{3}$, el desarrollo de las regiones rurales ${ }^{4}$ y el medio ambien$\mathrm{te}^{5}$. Los CCC, desde la venta de puerta en puerta, puestos de ventas en ferias de agricultores locales, cooperativas de consumo, redes informales de intercambio y comercialización de productos entre los vecinos y venta a comercios minoristas y restaurantes de la localidad hasta la distribución de los productos de la agricultura familiar en escuelas, etc., ganan mayor visibilidad social y catalizan movimientos sociales relacionados con la reconstrucción socioterritorial y comunitaria en todo el Estado, especialmente desde el comienzo del nuevo milenio, con lo que apoyan a los procesos de radicación territorial de las economías locales. En este caso, los factores de confianza y cercanía social se imbrican estrechamente con el factor productivo en la construcción socioterritorial.

Por su parte, el enfoque holístico de los SIAL facilita un tratamiento flexible y constructivo del territorio, que es visto como un espacio de proximidades, conformado por relaciones de mercado (sociedad) pero también por formas de cooperación derivadas de la confianza (comunidad) (Pecqueur, 1992).

Quizá la ventaja distintiva de los SIAL a la hora de facilitar los procesos de anclaje territorial sea el hecho de que, además de poner de relieve los elementos organizacionales en el territorio, sus interrelaciones, destacan el elemento «recurso local» como estrategia en torno a la que «anclar» el sistema productivo al territorio. Este enfoque territorial puede llegar a acotar, en la práctica, por medio de normativas y regulaciones, la producción local. En el caso de las DO se observa el mismo efecto, puesto que sus reglas pueden impedir la absorción de la producción local por parte de la gran industria. Por ejemplo, en el caso del queso comté, la DO regula unos métodos de producción de leche y elaboración de quesos que desalientan a las grandes compañías y fortalecen con ello la estructura productiva local. La ganadería se limita a una vaca por hectárea de pasto, la leche debe ser recogida dentro de un radio de $25 \mathrm{~km}$ y es necesaria la agrupación de los productores de leche, puesto que una rueda de queso requiere para su elaboración 450 litros, lo que genera cinco veces más empleos que la producción de emmental, a los que se suman, asimismo, los empleos vinculados al turismo gastronómico y patrimonial. Esto explica las tasas de migración rural del Franco Condado, mucho más bajas que en otras regiones rurales francesas. Además de fijar población, manteniendo unos niveles de renta muy superiores a los de otros espacios rurales, la DO Queso Comté

3. Bowen (2007), Menapace y Moschini (2012), Scarpa et al. (2005), Herrman y Teuber (2011), Josling (2006) y Deselnicu et al. (2013).

4. Bessiere (1998); Roest y Menghi (2000).

5. Bérard y Marchenay (2006), Riccheri et al. (2006). 
ha tenido un efecto positivo en el medio ambiente local, ya que ha ayudado a mantener la diversidad de flora porque granjeros y productores vinculan la calidad de la leche a la diversidad de especies vegetales que forman la dieta del ganado (Colinet et al., 2006).

Algo similar ocurre en la provincia de Santiago Rodríguez en la República Dominicana, caso analizado recientemente por Ibánez (2015), quien afirma que las barreras comerciales generan incentivos para el desarrollo rural y obligan a la cooperación entre las compañías procesadoras de leche y los pequeños granjeros. Las restricciones a la importación de alimentos han obligado a Nestlé a establecer una filial nacional para obtener leche local. Con el tiempo, la asociación local ha adquirido capacidades organizativas e institucionales considerables al ponerse al servicio de los pequeños agricultores y ganaderos y defender sus intereses.

Lo colectivo emerge como un elemento fortalecedor del producto local frente a los embates homogeneizadores de la industria. La concertación entre los actores es fundamental en los productos locales protegidos y se deben acordar métodos de producción, normas de calidad y control y para la distribución y comercialización de los productos.

\section{Conclusiones}

El papel de las agroalimentarias en el desarrollo rural se articula sobre su capacidad para promover una doble dinámica: la cualificación del producto y la cualificación del espacio rural. Más allá, el significado actual de los productos agroalimentarios de calidad, las redes alimentarias alternativas y los CCC plantean nuevas formas de abordar el desarrollo rural y la relación campo-ciudad.

Después de la gran ruptura agricultura-territorio, los productos agroalimentarios de calidad, diferenciados por su identificación geográfica, se presentan como una oportunidad de recomponer el vínculo, en una sociedad más sensible, con una manera de producir y consumir sostenible. Proporcionan una estrategia para que los agricultores puedan permanecer en la tierra y para que los productores puedan recuperar algo de poder y control sobre sus relaciones productivas.

La crisis ambiental a la que ha conducido el modelo productivista ha favorecido la floración de nuevas ideas para los modelos de desarrollo local, ahora vistos como sistemas complejos en transición. Entre otros, destacan los conceptos de «regiones socialmente innovadoras», «resiliencia» y "capacidad adaptativa», que requieren de análisis exhaustivos para ver cómo se materializan en la construcción territorial.

Las formas innovadoras de la oferta y la gestión de la demanda son necesarias para consolidar el mercado de los productos alimenticios locales y, en general, para convertir la retórica política contemporánea sobre agroalimentación sostenible y desarrollo rural en práctica.

La agricultura aún seguirá influyendo durante mucho tiempo en las construcciones de los territorios rurales. Las estructuras agrarias familiares que difieren de los sistemas basados en una lógica puramente productivista constituyen 
una ventaja para promover los productos de calidad vinculados al territorio. Permiten mantener las poblaciones y desarrollar un tejido social y económico favorable al territorio rural. Otras formas, con predominio de explotaciones profesionales y especializadas, pueden obstaculizar la recomposición de la relación entre la ciudad y el campo y el desarrollo territorial.

El desarrollo territorial debe estar basado en la complementariedad de las áreas rurales y urbanas, lo que requiere de áreas rurales con un tejido social cohesionado, con infraestructuras adecuadas, con sistemas transparentes y legítimos de gobernanza local, con un sistema económico diversificado y que dé soporte a muchas actividades productivas al mismo tiempo que promueva el respeto y la protección de sus valores ambientales. La vinculación de las áreas rurales con las urbanas es una muestra de cómo la cooperación y la sinergia entre grupos sociales y económicos, especialmente entre productores y consumidores, pueden apoyar el desarrollo rural sostenible y la puesta en marcha de modelos de producción y consumo más equilibrados, que contribuyen a evitar el riesgo de exclusión de los productores agrícolas sometidos a unas dinámicas globales cada vez más competitivas.

En el caso concreto del centro peninsular, cabe preguntarse qué aporta el modelo agrícola dominante a sus zonas rurales en retroceso demográfico, económico y social, y buscar opciones que permitan la dinamización de estos territorios.

\section{Referencias bibliográficas}

Acampora, Teresa y Fonte, María (2007). «Productos típicos, estrategias de desarrollo rural y conocimiento local». Opera, 7, 191-212.

AdAm, Alejandro (1828). Tratado de las antigüedades romanas. <https://books.google. es/books?id=AmGYDlGRUoMC $>$.

BAILEY, Robert (2011). Cultivar un futuro mejor: justicia alimentaria en un mundo con recursos limitados. Oxfam Internacional.

BecCATini, Giacomo (1979). «Dal settore industriale al distretto industriale. Alcune considerazioni sull'unitá di indagine dell'economia industriale». Rivista di Economia e Politica Industriale, 5 (1), 7-21.

Bérard, Laurence y MarchenaY, Philippe (2006). «Local Products and Geographical Indications: Taking Account of Local Knowledge and Biodiversity». International Social Science Journal, 58 (1), 109-16.

Bessiere, Jacinthe (1998). «Local Development and Heritage: Traditional Food and Cuisine as Tourist Attractions in Rural Areas». Sociologia Ruralis, 38 (1), 21-34.

Boucher, François y Muchnik, José (1998). Les agro-industries rurales en Amérique latine. Montpellier: CIRAD.

BoulANGer, Sylvaine y Legouy, François (dir.) (2008): «Vins, vignes et vignerons en France et dans le monde». Historiens et Géographes. dosier especial, 404 (mayo/ noviembre). Territories du vin [en línea], $\mathrm{n}^{\circ} 1$. «Pour une redéfinition des terroirs», 9 de diciembre de 2008. <http://revuesshs.u-bourgogne.fr/territoiresduvin/document.php?id=318 ISSN 1760-5296>.

Bowen, Sarah (2015). Divided Spirits. Tequila, Mezcal, and the Politics of Production. Oakland: University of California Press. 
Calle Collado, Ángel; Soler Montiel, Marta; Vara Sánchez, Isabel (2009). «La desafección al sistema agroalimentario: ciudadanía y redes sociales». En: I Congreso Español de Sociología de la Alimentación. Gijón 28 y 29 de mayo de 2009.

Campbell, Hugh (2009). «Breaking new ground in food regimes theory: Corporate environmentalism, ecological feedbacks, and the 'food from somewhere' regime». Agriculture and Human Values, 26 (4), 309-319.

Canto Fresno, Consuelo del; Ramírez García, Susana (2009). «Développement territorial en zones rurales métropolitaines: rôle des produits agroalimentaires de qualité dans la région de Madrid». Options méditerranéennes, série a: séminaires méditerranéens, 89, 255-270.

CAppechi, Vittorio (1987). «Formation professionnelle et petite entreprise: le développement industriel à spécialisation flexible en Emilie-Romagne», Revue Formation et Emploi, 19, 3-18.

CEPAL (2014). Agricultura familiar y circuitos cortos Nuevos esquemas de producción, comercialización y nutrición. Memoria del seminario sobre circuitos cortos realizado el 2 y 3 de septiembre de 2013. Santiago de Chile

Ciolos, Dacian (2012). «Agriculturas locales y circuitos de comercialización cortos: reforzar la dimensión local de la Política Agrícola Común». Conferencia Agricultura Local y Circuitos de Comercialización Cortos. SPEECH/12/283.

Colinet, Pierre; Desquilbet, Marion; Hassan, Daniel; Monier-Dilhan, Sylvette; Orozco, Valérie and Réquillart, Vincent (2006). Case Study: Comté Cheese in France. Toulouse: Institut Nationale de la Recherche Agronomique, Unité d'Economie et Sociologie Rurales.

Colonna, Paul; Fournier, Stéphane; Touzard, Jean Marc; Abécassis, Joel; Broutin, Cécile; Chabrol, Didier; Champenois, Armelle; Deverre, Christian; François, Martine; Lo Stimolo, Danielle; Méry, Vanessa; Moustier, Paule y Trystram, Gilles (2013). «Food systems». En: Esnouf, Catherine; Russel, Marie y Bricas, Nicolás (eds.). Food system sustainability: Insights from duALIne. Cambridge: Cambridge University Press, 69-100.

Courlet, Claude; Soulage, Bernard y Pecqueur, Bernard (1993). "Industrie et dynamiques de territoires». Revue d'Économie Industrielle, 64 (2), 7-21.

Deselnicu, Oana; Costanigro, Marco; Souza-Monteiro, Diogo y McFadden, Dawn Thilmany (2013). "A Meta-Analysis of Geographical Indication Food Valuation Studies: What Drives the Premium for Origin-Based Labels?». Journal of Agricultural and Resource Economics, 38 (2).

Freitas Caetano, Silvia; Ramírez García, Susana; Canto Fresno, Consuelo del (2013). «Red de relaciones, cohesión social y dinámica territorial. El caso de la Denominación de Origen de Vinos de Uclés (Cuenca)». En: Rodríguez MartíNEZ, Francisco (coord.). Desarrollo local en tiempos de crisis: ¿El retorno a los recursos endógenos? Granada: Universidad de Granada, 209-221.

Friedmann, Harriet y McMichael, Philip (1989) «Agriculture and the State System: the Rise and Decline of National Agricultures, 1870 to the Present». Sociologica Ruralis, 29 (2), 93-117.

Fukuoka, Masanobu (2009). The One-Straw Revolution: an introduction to natural farming. Nueva York: New York Review of Books.

GonzÁlez de Molina, Manuel (2012). «Agroecología y políticas públicas en Europa». Agroecología, 6, 75-88.

GIS-SYAL (2009). Bilan du Groupement d'Intérêt Scientifique Systémes Agroalimentaires Localisés (2001-2007). 
Grupo ETC (2009). «¿Quién nos alimentará? Preguntas sobre las crisis alimentaria y climática». Communiqué, 102.

Herrman, Roland y Teuber, Ramona (2011). "Geographically differentiated products». En: Lusk, Jayson L.; Roosen, Jutta y Shogren, Jason F. (eds.). The Oxford Handbook on the Economics of Food Consumption and Policy. Oxford: Oxford University Press, 811-842.

IвÁÑEZ, Lindsey (2015). «Beyond inclusiveness: institutions, cooperation and rural development». Canadian Journal of Development Studies / Revue Canadienne d'Études du Développement, 36 (4), 499-515.

IICA (2013). Sistemas Agroalimentarios Localizados (SIAL), una nueva visión de gestión territorial en América Latina: experiencias en territorios de Argentina, Costa Rica, Ecuador y México. México: IICA, CIRAD.

IINUMA, Jiro (1995). Japanese farming: past and present. Tokio: Nobunkyo.

Ilbery, Brian y Bowler, Ian (1998). «From Agricultural Productivism to Postproductivism». En: Ilbery, Brian (ed.). The Geography of Rural Change. Londres, 57-84.

JANVRY, Alain de y SAdoulet, Elisabeth (2005). "Achieving success in rural development: toward implementation of an integral approach». Agricultural Economics, 32 (1), 75-89.

Josling, Tim (2006). "The War on Terroir: Geographical Indications as a Transatlantic Trade Conflict». Journal of Agricultural Economics, 57 (3), 337-363.

Kerr, William (2006). «Enjoying a Good Port with a Clear Conscience: Geographic Indicators, Rent Seeking, and Development». The Estey Center Journal of International Law and Trade Policy, 7, 1-14.

Kneafsey, Moya; Cox, Rosie; Holloway, Lewis; Dowler, Elizabeth; Venn, Laura y Tuomainen, Helena (2008). Reconnecting Consumers, Producers and Food. Exploring Alternatives. Oxford y Nueva York: Berg.

Longe, Marcia S. de; Miles, Albie y Carlisle, Liz (2016). «Investing in the transition to sustainable agriculture». Environmental Science \& Policy, 55, 266-273.

López García, Daniel (2012). Hacia un modelo europeo de Extensión Rural Agroecológica. Praxis participativas para la transición agroecológica. Un estudio de caso en Morata de Tajuña, Madrid. Baeza: Universidad Internacional de Andalucía. Tesis doctoral.

López, Elisabeth y Muchnik, José (coord.) (1997). Petites entreprises et grands enjeux: le développement agroalimentaire local. París: L'Harmattan.

Magrama (2OI2). Canales cortos de comercialización en el sector agroalimentario. Madrid: Ministerio de Agricultura, Alimentación y Medio Ambiente.

Mancha Cáceres, Olga y Ramírez García, Susana (2014). «Propuestas agroecológicas para un desarrollo sociocomunitario alternativo en Guadalajara. Periferias, fronteras y diálogos». Actas del XIII Congreso de Antropología de la Federación de Asociaciones de Antropología del Estado Español. Tarragona: Universitat Rovira i Virgili, 2643-2665.

Manhire, Jon; Moller, Hans; Barber, Andrew; Saunders, Caroline; MacLeod Catriona; Rosin, Chris; Lucock, Dave; Post, Elizabeth; Ombler, Franz; Campbell, Hugh; Benge, Jayson; Reid, John; Hunt, Lesley; Hansen, Paul; Carey, Peter; Rotarangi, Stephanie; Ford, Stuart; Barr, Tremane (2012). «The New Zealand Sustainability Dashboard: Unified monitoring and learning for sustainable agriculture in New Zealand». ARGOS Working Paper 8.

Marsden, Terry (2004). "Theorizing Food Quality: Some Issues in Understanding its Competitive Production and Regulation». En: Harvey, Mark, McMeekin, Andrew y Warde, Alan (eds). Qualities of Food. Manchester: Manchester University Press. 
Marsden, Terry; Sonnino, Roberta (2008). «Rural development and the regional state: Denying multifunctional agriculture in the UK». Journal of Rural Studies, 24, 422-431.

McMichael, Philip (2002). «La restructuration globale des systems agro-alimentaires». Mondes en Developpement, 30 (117), 45-54.

Menapace, Luisa y Moschini, Gino Carlo (2012). "Quality certification by geographical indications, trademarks and firm reputation». European Review of Agricultural Economics. 39 (4), 539-566.

Milone, Pierluigi (2014). «Agricultura campesina y circuitos cortos: nuevas tendencias en Europa». En: Agricultura familiar y circuitos cortos. Nuevos esquemas de producción, comercialización y nutrición. Santiago de Chile: CEPAL.

Mollard, Amédée (2001). "Qualité et développement territorial: une grille d'analyse théorique à partir de la rente». Économie Rurale, 263 (1), 16-34.

Monllor, Neus (2013). «El nuevo paradigma agrosocial, futuro del nuevo campesinado emergente». Polis, 12 (34), 203-223.

Montasell Dorda, Josep; Callau Berenguer, Sonia y Vila Pascual, Andreu (2014). "Células alimentarias. Un nuevo concepto para re-pensar las prácticas tradicionales de planificación alimentaria. El caso de la región metropolitana de Barcelona». En: Pavón Gamero, David; Ribas Palom, Anna; Ricart Casadevall, Sandra; Roca Torrent, Anna; Salamaña Serra, Isabel y Tous de Sousa, Cristina (eds.). XVII Coloquio de Geografía Rural. COLORURAL. Revalorizando el espacio rural: leer el pasado para ganar el futuro. Girona: Documenta Universitaria, 651-668.

Muchnik, José y SAutier, Denis (1998). Proposition d'action thématique programmée: systémes agroalimentaires localisés et construction de territoires. Montpellier: CIRAD.

Muramoto, Joji; Hidaka, Kazumasa y Mineta, Takuya (2010). "Japan: finding opportunities in the current crisis». En: Gliessman, Stephen R. y Rosemeyer, Martha. The conversion to sustainable agriculture: principles, processes, and practices. Taylor \& Francis Group, 273-301. <https://goo.gl/Yqpph4>.

Pecqueur, Bernard (1992). «Territoire, territorialité et développement». En: Industries et territoires: les systèmes productifs localisés. Actes du colloque de Grenoble, IREPD, 71-88.

- (2001). «Qualité et développement territorial: l'hypothèse du panier de biens et de services territorializés». Économie Rurale, 261, 37-49.

PÉrez, Edelmira (2001): «Hacia una nueva visión de lo rural». En: GiarracCA, Norma (coord.). ¿Una nueva ruralidad en América Latina? Buenos Aires: CLACSO, 17-29.

PImbert, Michel (2008). Hacia la soberanía alimentaria: recuperación de sistemas autónomos de alimentos. Sussex: IIED.

Ploeg, Jan Douwe van der (2008). Los agricultores y los imperios de alimentos, las luchas por la autonomía y la sostenibilidad en la era de globalización. Porto Alegre: UFRGS.

- (2010). Nuevos campesinos, campesinos e imperios alimentarios. Barcelona: Icaria.

Ploeg, Jan Douwe van der y Marsden, Terry (2008). Unfolding Webs: The Dynamics of Regional Rural Development. Assen (Países Bajos): Van Gorcum.

Porter, Michael (1998). "Clusters and the new economics of competition». Harvard Business Review (noviembre-diciembre de 1998).

Renting, Henk; Rossing, Walter A. H.; Groot, Jeroen. C. J.; Ploeg, Jan Douwe van der; Laurent, Catherine E.; Perraud, Daniel; Stobbelaar, Derk Jan; Ittersum, Martin K. van (2009). "Exploring multifunctional agriculture. A review of conceptual approaches and prospects for an integratie transtional framework». Journal of Environmental Management, 90, suplemento 2, 112-123. 
Riccheri, Mariano; Görlach, Benjamín; Schegel, Stephanie; Keefe, Helen y LeipPRAND, Anna (2006). Assessing the Applicability of Geographical Indications as a Means to Improve Environmental Quality in Affected Ecosystems and the Competitiveness of Agricultural Products. EC WP3 Project on Impacts of the IPR Rules on Sustainable Development. Ecologic Institute. <http://ecologic.eu/sites/files/ download/projekte/1800-1849/1802/wp3_final_report.pdfs.

Roest, Kees de y Menghi, Alberto (2000). «Reconsidering 'Traditional' Food: The Case of Parmigiano Reggiano Cheese». Sociologia Ruralis, 40: 439-451.

Romero, Guiselle (2014). «Agricultura familiar, circuitos cortos y el Programa Nacional de Alimentación Escolar Qali Warma, Perú». En: Agricultura familiar y circuitos cortos: Nuevos esquemas de producción, comercialización y nutrición. Memoria del seminario sobre circuitos cortos, realizado el 2 y 3 de septiembre de 2013. Santiago de Chile: CEPAL.

Sabourin, Eric; Samper, Mario y Sotomayor, Octavio (coord.) (2014). Políticas públicas y agriculturas familiares en América Latina y el Caribe: balance, desafíos y perspectivas. Santiago de Chile: CEPAL.

Scarpa, Riccardo; Philippidis, George y Spalatro, Fiorenza (2005). «Productcountry images and preference heterogeneity for Mediterranean food products: A discrete choice framework». Agribusiness, 21 (3), 329-349.

Schejtman, Alexander y Berdegué, Julio A. (2004). Desarrollo territorial rural. Santiago de Chile: Rimisp - Centro Latinoamericano para el Desarrollo Rural.

Schmitz, Hubert (1996). "Efficacité collective: chemin de croissance pour la petite industrie dans les pays en développement». En: Pecqueur, Bernard (dir.). Dynamiques territoriales et mutations économiques. París : L'Harmattan, 73-99.

- (1997). "Collective efficiency and increasing returns». IDS Working Paper, 5.

Shucksmith, Mark (1993). «Farm household behaviour and the transition to postproductivism». Journal of Agricultural Economics, 44, 466-478.

Soler Montiel, Marta; Calle Collado, Ángel (2010). «Rearticulando desde la alimentación: canales cortos de comercialización en Andalucía». En: Soler MoNtiel, Marta y Guerrero Quintero, Carmen. Patrimonio cultural en la nueva ruralidad andaluza. Sevilla: PH Cuadernos, Junta de Andalucía. Consejería de Cultura, 259-283.

Torjusen, Hanne; Lieblein, Geir y Vitters $\varnothing$, Gunnar (2008). «Learning, communicating and eating in local food-systems: The case of organic box schemes in Denmark and Norway». Local Environment, 13 (3): 219-234.

Trubek, Amy (2009). The Taste of Place: A Cultural Journey into Terroir. Berkeley: University of California Press.

Vivas, Esther (2014). El negocio de la comida. ¿Quién controla nuestra alimentación? Barcelona: Icaria.

Wiskerke, Johannes. S. C. (2009). «On places lost and places regained: Reflections on the alternative food geography and sustainable regional development». International Planning Studies, 14 (4), 369-387. 